Copyright by the Acoustical Society of America. Guigou, C., Fuller, C. R., \& Wagstaff, P. R. (1994). Active isolation of vibration with adaptive structures. Journal of the Acoustical Society of America, 96(1), 294-299. doi: 10.1121/1.411439

\title{
Active isolation of vibration with adaptive structures ${ }^{\text {a) }}$
}

\author{
C. Guigou and C. R. Fuller \\ Vibration and Acoustics Laboratories, Mechanical Engineering Department, Virginia Polytechnic Institute \\ and State University, Blacksburg, Virginia 24061-0238 \\ P. R. Wagstaff \\ Département Génie Mécanique, Division Acoustique et Vibration, Université de Technologie de Compiègne, \\ B.P. 649, 60206 Compiène Cedex, France
}

(Received 29 September 1992; revised 21 October 1993; accepted 14 December 1993)

\begin{abstract}
The problem of actively isolating the periodic vibrations of a rigid machine mounted on a supporting flexible structure is usually approached by applying the active inputs in parallel or series with the passive inputs. This has a number of disadvantages which are related to the development of a high power, compact yet stiff/active isolation unit. In this experimental work, a new approach in which the receiving structure is considered to have adaptive properties is studied. The aim is to control the transmitted vibrations by distributed arrays of piezoelectric transducers bonded to the receiving structure. The experimental rig consists of a rigid thick plate (the machine) supported at the corners by four elastic springs mounted on a thin clamped-free elastic steel plate (the receiving structure). The thick plate is driven by a harmonic force input. Response in the receiving panel is measured with a scanning laser vibrometer. Active inputs to the receiving structure are induced by three pairs of piezoceramic actuators bonded to the surface and configured to induce bending. The error sensors consist of up to two polyvinylidene fluoride (PVDF) strips attached to the panel surface in various positions. The control approach uses a two channel feedforward adaptive LMS algorithm implemented on a TMS320C25. The results show that the first three modes of the system can be controlled efficiently when driven "on resonance," thus effectively isolating the vibrating structure from the "machine" raft input. However, when the system is driven "off resonance," the vibrations of the receiving structure proved more difficult to reduce effectively. The paper presents vibration distribution of the receiving plate with and without control for a number of input frequencies as well as a variety of control transducer configurations.
\end{abstract}

PACS numbers: $43.40 . \mathrm{Vn}$

\section{INTRODUCTION}

The problem of isolating the periodic vibrations of a rigid machine mounted on a supporting flexible structure was solved in the past by using a passive approach, which was shown to be inefficient at low frequencies. Advances in electronics, digital signal processors, and control theory suggest the possibility of using active or combined active-passive techniques. These techniques provide better results at low frequencies. An overview of the present active techniques of vibration isolation has been presented by Von Flotow. ${ }^{1}$ Active inputs are usually applied either in parallel or in series with the passive inputs. This has a number of disadvantages that are related to the development of high power, compact, yet stiff passive-active isolation units. Nelson $e a^{2} .^{2}$ implemented a multiple parallel active-passive mounting system. The resulting isolation system was shown to be especially effective at resonant frequencies of the supporting structure. However, in cases of off-resonant frequencies, considerable care has to be taken to prevent an increase of the vibration level. Other experiments on the same kind of rig (activepassive mounting system) have been conducted by Lefebvre, ${ }^{3}$ who used polyvinylidene fluoride (PVDF) sensors

a) This paper was first presented in brief form in the proceedings of NOISECON 91. to provide information on structural vibrations to the control system. This type of sensor has been shown to be relevant, even though the effectiveness of the control itself is highly dependent on the location of these sensors. In Lefebvre's experiment as in that of Nelson et al., a high attenuation level was obtained for on-resonance excitation but poor results were obtained for off-resonance frequencies.

In the experiment described here, a different approach to vibration isolation is studied. The aim is to control the transmitted vibrations by distributed arrays of piezoelectric transducers. These transducers are bonded to the supporting structure, and hence become an integral part of it. This type of structure is labeled "adaptive structure" because the actuators can change the system properties in a controlled manner. Distributed PVDF piezoelectric strips attached to the receiving structure in various positions are used as error sensors. They provide the error signals to be reduced during the control process. The controlled structure then results from the combination of both sensors and actuators integrated with the supporting structure.

The control approach uses a two-channel feedforward adaptive LMS algorithm implemented on a TMS320C25. The principle of the active control technique is to minimize the error signals emitted by the sensors by applying control signals through the secondary active sources, which are the piezoelectric actuators. 


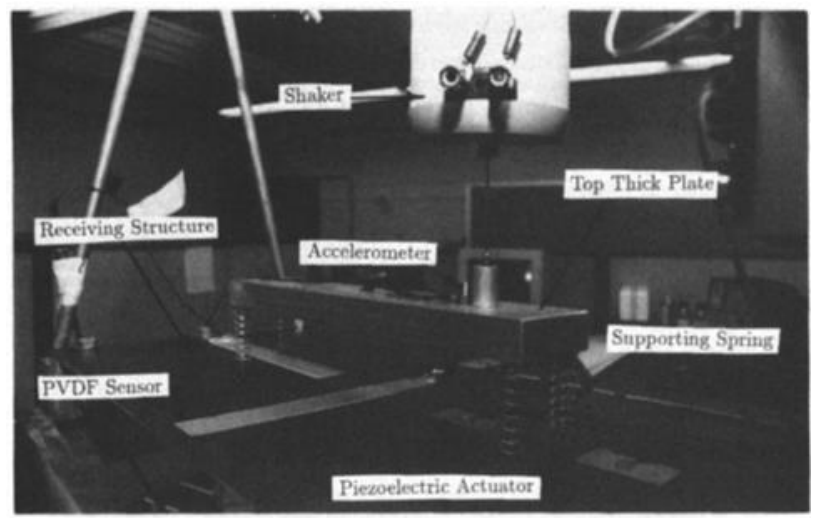

FIG. 1. Experimental setup.

In conducting the tests, different cases were studied for isolating vibrations due to a disturbance for both on- and off-resonance frequencies. In addition, the number of control channels was varied to illustrate the advantages of distributed control.

\section{EXPERIMENTAL SETUP}

The experimental setup, shown in Fig. 1, consists of a rigid thick plate (the machine) supported at the corners by four elastic springs mounted on a thin clamped-free elastic steel plate (the supporting or receiving structure). The upper thick plate is made of aluminum and measures $267 \times 50 \times 19$ $\mathrm{mm}$. The stiffness of the springs is $0.137 \mathrm{~kg} / \mathrm{mm}$. The receiving plate is $357 \times 255 \times 1.5 \mathrm{~mm}$ steel plate. The clamped boundary conditions (on two opposite edges of the receiving plate) are achieved by three screws along each boundary connecting the receiving plate to a rigid, heavy steel frame.

The thick plate is excited by a harmonic force input, i.e., is driven with a shaker attached to the structure with a stinger. The stinger is located at about one-fourth of the length from the edge and in the middle of the width of the upper plate. A Bruel and Kjaer accelerometer is also positioned near the stinger on the thick plate to measure the vibration amplitude variation when the control is applied.

The receiving plate is configured with three pairs of piezoelectric actuators. Each actuator consists of two piezoelectric elements of dimensions $38.1 \times 21 \times 0.19 \mathrm{~mm}$ bonded symmetrically on each side of the plate. All piezoelectric elements are bonded to the structure with M-bond 200 adhesive. The symmetrically located patches are connected outof-phase such that a voltage which induces extension on one side of the plate would induce contradiction on the opposite side, resulting in uniform bending about the neutral axis of the plate. With this configuration, driving the actuator electrically results in line moments acting around the boundaries of the piezoelectric transducer, as shown by Dimitriadis et $a l^{4}$ The actuators may be located either to control particular modes of vibration of the receiving structure or to directly, oppose the input forces at the mounting points. For the experiments described, the modal approach was used in order to limit the number of actuators.

Distributed PVDF error sensors of dimensions $155 \times 22$ $\mathrm{mm}$ are also attached in various positions to the receiving plate with double-sided tape to provide a measure of the plate response. The PVDF strip is a long chain, semicrystalline polymer of the repeated unit $\mathrm{CH}_{2}-\mathrm{CF}_{2}$, which produces an electrical charge per unit electrode area across the thickness of the polymer due to the stress applied along the transverse axis. Since the PVDF output voltage is proportional to the strain, the PVDF location has to be chosen carefully. The distributed PVDF strip in effect integrates the local charge generated by local strain deformation. In other words, the output voltage of the PVDF strip is proportional to the integral of the surface strain. For a PVDF strip of constant polarization and of rectangular shape covering a surface $S$, the closed circuit charge is ${ }^{5}$

$$
q=-z_{0} \int_{S}\left(e_{31} \frac{\partial^{2} w}{\partial x^{2}}+e_{32} \frac{\partial^{2} w}{\partial y^{2}}+2 e_{36} \frac{\partial^{2} w}{\partial x \partial y}\right) d x d y
$$

where $z_{0}$ is the $z$ coordinate of the PVDF layer midplane; $e_{31}, e_{32}, e_{36}$ are the piezoelectric stress/charge constants and $w(x, y)$ is the out-of-plane displacement of the structure. Then, it can be noted that the value of Eq. (1) becomes zero if $w(x, y)$ is an odd function with respect to the integration domain $S$, or if $w(x, y)$ is a constant function on the same domain. It is thus possible for the PVDF strip to have a net output charge of zero even though the plate has significant vibratory motion. If the output voltage from the PVDF strip is zero (while the out-of-plane vibrations amplitudes are not) then no error signal is sent to the computer which makes control impossible. Therefore, the control efficiency depends closely on the capabilities of the PVDF to observe the correct response of the receiving plate and then to transmit response information through its output voltage.

\section{DESCRIPTION OF THE CONTROLLER}

To achieve control, a two-channel adaptive controller based on the multichannel version of the Widrow-Hoff filtered-X control algorithm was implemented on a TMS320C25 DSP resident in an AT computer. This algorithm is fully described by Elliot et al. ${ }^{6}$

A block diagram illustrating the control system is presented in Fig. 2. The output of an error sensor can be represented at the $n$th time step by

$$
\begin{aligned}
e_{l}(n)= & d_{l}(n)+\sum_{m=1}^{M} \sum_{j=0}^{N-1} P_{l m j} \sum_{i=0}^{N-1} w_{m i}(n-j) \\
& \times x(n-i-j),
\end{aligned}
$$

where $d_{l}(n)$ is the $l$ th error sensor output due to the input excitation alone, $x(n)$ is the input reference source, $w_{m i}$ are the coefficients of the adaptive FIR filters and $\boldsymbol{P}_{\boldsymbol{l m} j}$ is the $j$ th coefficient of the transfer function between the output of the $m$ th adaptive filter and the $l$ th error sensor. $M$ and $N$ are, respectively, the number of control actuators and filter coefficients. In the LMS algorithm, the error function is defined by the mean square error signal; i.e.,

$$
J=E\left(\sum_{l=1}^{L} e_{l}^{2}(n)\right)
$$




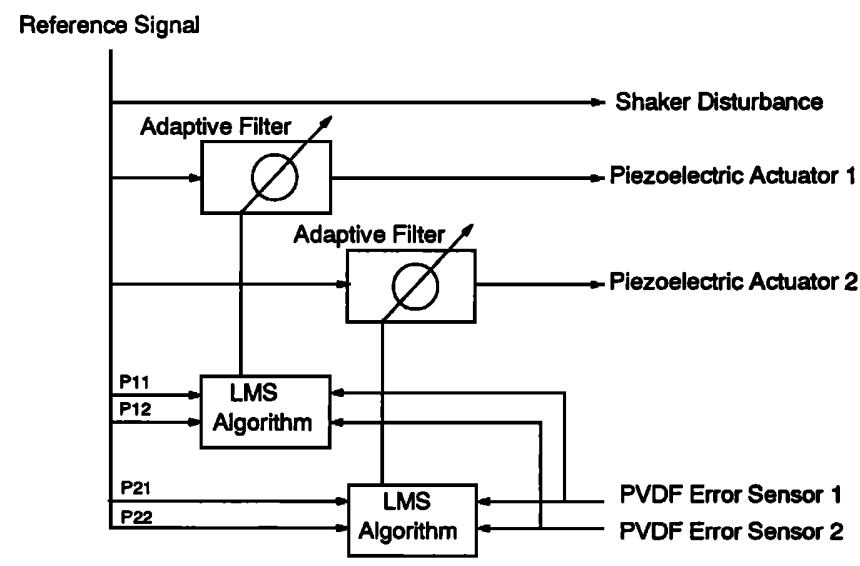

FIG. 2. Block diagram of controller.

where $E$ is the expectation operator and $L$ is the total number of error sensors utilized to implement the control. Since this error function is quadratic, only one minimum solution exists.

At each sample time $n$, the outputs of the compensating filters $\boldsymbol{P}_{l m j}$ are used by the LMS algorithm to minimize $\boldsymbol{J}$ by individually updating each of the adaptive filter coefficients according to the relation

$$
w_{m i}(n+1)=w_{m i}(n)-\mu \sum_{l=1}^{L} e_{l}(n) r_{l m}(n-i),
$$

where the outputs of the compensating filters $\boldsymbol{P}_{l m j}$ are

$$
r_{l m}(n-i)=\sum_{j=0}^{N-1} P_{l m j} x(n-i-j),
$$

and $\mu$ is the stability parameter.

For all tests conducted, a steady-state sinusoid is used as the disturbance signal. A Wavetek model 275 signal generator is used to create this disturbance and the reference signal for the LMS controller. Depending on the number of error sensors used, either one of two control channels are implemented for the tests, as in the time-domain version LMS algorithm the number of error sensors has to be greater than or equal to the number of control channels $(L \geqslant M)$ and the experiments are limited to two PVDF sensor strips.

\section{EXPERIMENTAL PROCEDURE}

A modal analysis was first performed in order to determine the resonant frequencies of the system. First, a "tap test" was conducted on the receiving plate (without the springs and the top plate) to verify if the clamped-free boundary conditions were modeled. Table I presents the six first theoretical (computed from Leissa ${ }^{7}$ ) and experimental resonant frequencies of the receiving plate. It can be seen that measured resonant frequencies agree well with the theoretical ones. Table I also shows the expected mode shapes corresponding to these resonant frequencies.

When the thick top plate is attached by the four springs to the receiving plate, only the symmetric-symmetric and antisymmetric-symmetric modes, which do not have a nodal line passing through the attachment location of the springs, can be excited by the driving shaker. This is due to the sym-

\begin{tabular}{|c|c|c|c|}
\hline $\begin{array}{l}\text { Mode } \\
\text { Number }\end{array}$ & $\begin{array}{c}\text { Theoretical } \\
\text { Frequency } \\
(\mathrm{Hz})\end{array}$ & $\begin{array}{l}\text { Experimental } \\
\text { Frequency } \\
(\mathrm{Hz})\end{array}$ & $\begin{array}{l}\text { Mode } \\
\text { Shape }\end{array}$ \\
\hline 1 & 38 & 37 & + \\
\hline 2 & 60 & 58.5 & -- \pm- \\
\hline 3 & 108 & 112 & $+i-$ \\
\hline 4 & 140 & 136 & $\pm-\frac{1}{1}=$ \\
\hline 5 & 166 & 175 & $\begin{array}{l}-\frac{t}{t}-0 \\
-\bar{f}\end{array}$ \\
\hline 6 & 199 & 201 & $+i-i+$ \\
\hline
\end{tabular}

TABLE I. Resonant frequencies of receiving plate.

metry of the system with respect to the width. Thus, when the shaker drives the top plate, the three first modes of the global experimental setup are found to correspond to the frequencies $38.5,106$, and $176 \mathrm{~Hz}$, which have to be related to the first, third, and fifth resonant frequencies of the receiving plate, as shown in Table I. Then, when the receiving plate is coupled with the top plate, the global modal characteristics still match with those of the clamped-free plate.

For the tests, it was decided that the control be applied for these three resonant frequencies and for an arbitrary offresonance frequency of $85 \mathrm{~Hz}$.

The test procedure was identical for both on- and offresonance cases. Before applying the control, the out-ofplane velocity response of the receiving plate was measured with a scanning laser vibrometer to identify the shape of the response of the excited receiving structure. The advantage of using a scanning laser vibrometer is that it does not interfere with the system response. After obtaining measurements for the uncontrolled case, the filtered-X LMS algorithm was invoked, and the system was controlled via the piezoelectric actuators, using error information from the PVDF sensors. Upon converging to the minimum response, structural measurements were again obtained using the laser vibrometer. After obtaining these measurements, the uncontrolled and controlled responses were compared. The control effectiveness was then evaluated by measuring the amplitudes of the receiving plate obtained from the laser scan at a total of $N=30$ positions over the scanned surface of the receiving plate. The control effectiveness in $\mathrm{dB}$ is defined as

$$
E_{f}=10 \log _{10} \frac{\sum_{i=1}^{N} A_{i}^{2}(\text { before control })}{\sum_{i=1}^{N} A_{i}^{2}(\text { after control })}
$$

where $A_{i}$ is the amplitude of the receiving plate at the $i$ th position. The effectiveness $E_{f}$ is related to the attenuation in energy density of out-of-plane response of the structure. 
TABLE II. Results of isolation control.

\begin{tabular}{|c|c|c|c|c|}
\hline $\begin{array}{l}\text { Frequency } \\
\text { (Hz) }\end{array}$ & Configuration & $\begin{array}{l}\text { Error sensors } \\
\text { and actuators }\end{array}$ & $\begin{array}{c}\text { Effectiveness } \\
\text { (dB) }\end{array}$ & $\begin{array}{l}\text { Top plate } \\
\text { amplitude } \\
\text { variation } \\
\text { (dB) }\end{array}$ \\
\hline \multirow{3}{*}{38.5} & \multirow{3}{*}{ (a) } & $\mathrm{S} 1, \mathrm{C} 1$ & 17.8 & -0.5 \\
\hline & & $\mathrm{S} 1, \mathbf{S} 2, \mathrm{C} 1$ & 20.3 & -0.8 \\
\hline & & $\mathrm{S} 1, \mathrm{~S} 2, \mathrm{C} 1, \mathrm{C} 2$ & 24.0 & -3.5 \\
\hline \multirow{2}{*}{106.0} & \multirow{2}{*}{ (b) } & $\mathrm{S} 2, \mathrm{C} 1$ & 2.2 & +0.2 \\
\hline & & $\mathrm{S} 1, \mathrm{~S} 2, \mathrm{C} 1, \mathrm{C} 2$ & 30.9 & +0.8 \\
\hline \multirow{2}{*}{176.0} & \multirow{2}{*}{ (c) } & $\mathrm{S} 1, \mathrm{C} 1$ & -0.4 & -2.0 \\
\hline & & $\mathrm{S} 1, \mathrm{~S} 2, \mathrm{C} 1, \mathrm{C} 2$ & 14.0 & 0.0 \\
\hline 85.0 & (b) & $\mathrm{S} 1, \mathrm{~S} 2, \mathrm{C} 1, \mathrm{C} 2$ & 4.0 & 0.1 \\
\hline
\end{tabular}

In the legend of the figures and tables comparing results, " $\mathrm{C \# "} \mathrm{indicates} \mathrm{the} \mathrm{respective} \mathrm{piezoelectric} \mathrm{actuator} \mathrm{used} \mathrm{in}$ the control implementation, and "S\#" indicates the chosen PVDF error sensor.

\section{RESULTS}

For all the tests conducted, the results of the control effectiveness, the top plate amplitude variations and the various configurations for the sensor and actuator positions are summarized in Table II and Fig. 3, respectively.

For the first set of experiments, the top plate is excited at a frequency of $38.5 \mathrm{~Hz}$ corresponding to the first mode of the receiving plate. Due to the response of the receiving plate, the PVDF sensors need to be positioned parallel to the freeboundary conditions so that the sensors can give information about the mode shape to the controller. Figure 3 shows the configuration of actuators and sensors. Before control is applied, the mode shape of the supporting plate as shown in Fig. 4(a) corresponds to the one expected (as described in Table I), i.e., with a maximum of vibration in the central zone of the plate. When the control is applied, global attenuation of the vibration amplitude is obtained. Improvement in (a)

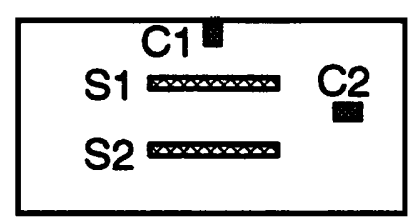

(b)

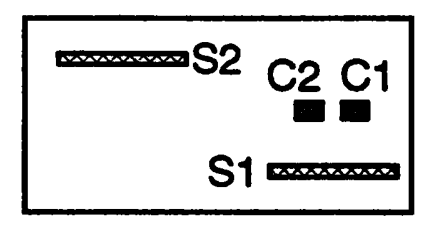

(c)

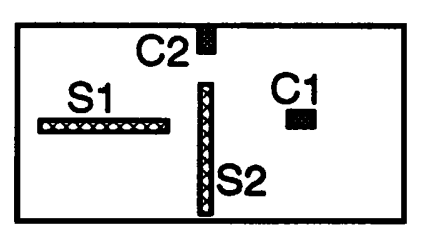

FIG. 3. Configuration of sensors and actuators on receiving plate.
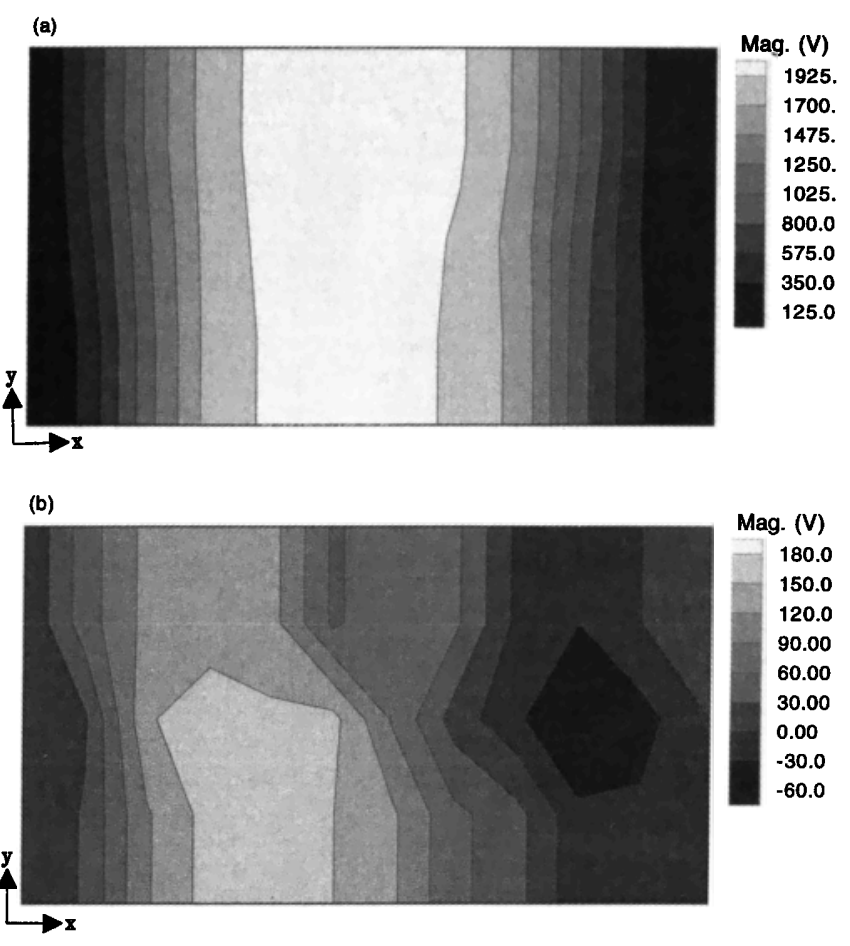

FIG. 4. Receiving plate vibration distribution at $38.5 \mathrm{~Hz}$. (a) Without control, (b) with control (S1,S2,C1,C2).

structural vibration energy density is observed when first the number of error sensors and then the number of control actuators are increased. When a single actuator and a single sensor are used to implement the control, the maximum vibration level obtained in the central zone of the receiving plate is reduced by a factor of 8 and a global vibration attenuation of $17.8 \mathrm{~dB}$ is achieved. The addition of a second sensor increases the control effectiveness with a global attenuation of $20.3 \mathrm{~dB}$. When two control channels are implemented (two actuators and two sensors), the control effectiveness is further increased to obtain a $24 \mathrm{~dB}$ reduction in vibration level. Figure 4(b) shows the vibration distribution of the receiving plate when the two channels are invoked. It can also be noticed that for these cases, the top plate vibration levels were slightly attenuated by the control.

The excitation frequency is $106 \mathrm{~Hz}$ for the second set of experiments. The vibration levels before the control is applied are shown in Fig. 5(a). In this case, as expected, a nodal line parallel to the clamped boundary conditions is located in the central zone of the receiving plate, dividing the plate into two parts vibrating out-of-phase. The vibratory motion is still almost invariant in the direction parallel to the clamped boundary conditions. Thus the PVDF sensors have to be positioned perpendicular to the clamped boundary conditions, as their output voltage is proportional to the strain. Note that if the PVDF sensors were symmetrically located accross the nodal line, their output voltage will be zero. When only one control channel is used, the vibration amplitudes are slightly decreased and the vibration pattern is unchanged [i.e., as a $(2,1)$ mode]. However, the output signal from the PVDF sensor is considerably attenuated (18-dB attenuation). This is due to the fact that the out-of-plane displacement becomes 


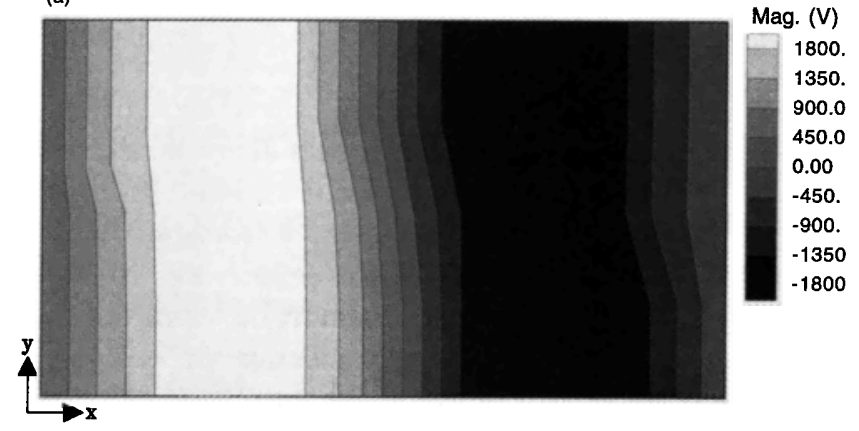

(b)

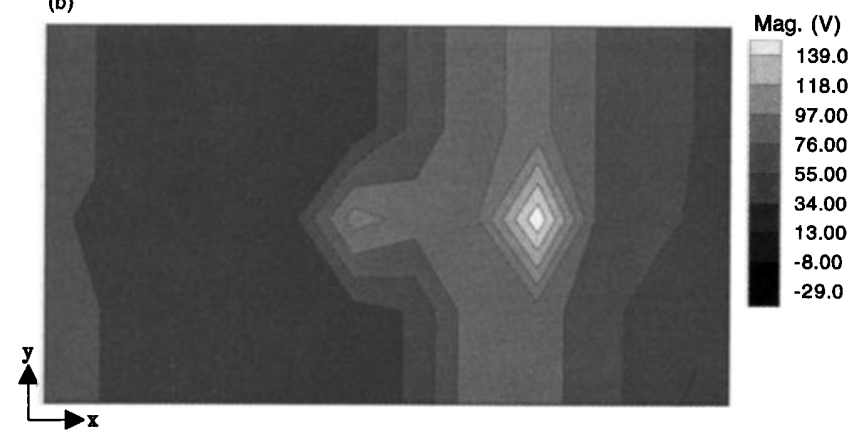

FIG. 5. Receiving plate vibration distribution at $106 \mathrm{~Hz}$. (a) Without control, (b) with control (S1,S2,C1,C2).

more constant on the surface of the PVDF sensor, i.e., the curvature of the receiving plate is reduced on the sensor surface. As explained in Sec. I, this causes a zero output charge from the sensor. Therefore, the control actuator acts in such a way that the output voltage of the PVDF strip is well attenuated even though the receiving plate still has significant vibration amplitude. This shows the disadvantage of PVDF distributed sensors, which may give an output voltage of zero while the out-of-plane vibration amplitudes are nonzero. However, the use of only one PVDF in this case does not provide enough information about the global mode shape of the receiving plate. Thus two PVDF located on each side of the nodal line are necessary to describe the motion of the plate. Figure 5(b) shows that, when two control actuators are applied to the system, the vibrations of the receiving plate are almost completely attenuated. Some residual vibration response is detected between the two actuators. For both controlled cases, the top plate vibration levels are slightly increased.

The third set of experiments corresponds to an excitation frequency of $176 \mathrm{~Hz}$. In this particular case, the receiving plate vibrates such that two nodal lines are positioned parallel to the free-boundary conditions as observed in Fig. 6(a). Then, the PVDF sensors can be placed either perpendicular or parallel to the free-boundary conditions, as the vibratory motion is no longer invariant in both the parallel and perpendicular directions to the free-boundary conditions. For one channel of control, even though the output voltage from the PVDF sensor is reduced by $11 \mathrm{~dB}$ and the top plate vibrations are decreased $(0.2 \mathrm{~dB})$, the receiving plate vibrations are slightly increased and distorted near the actuator position. However, the global vibration pattern was unchanged [i.e.,

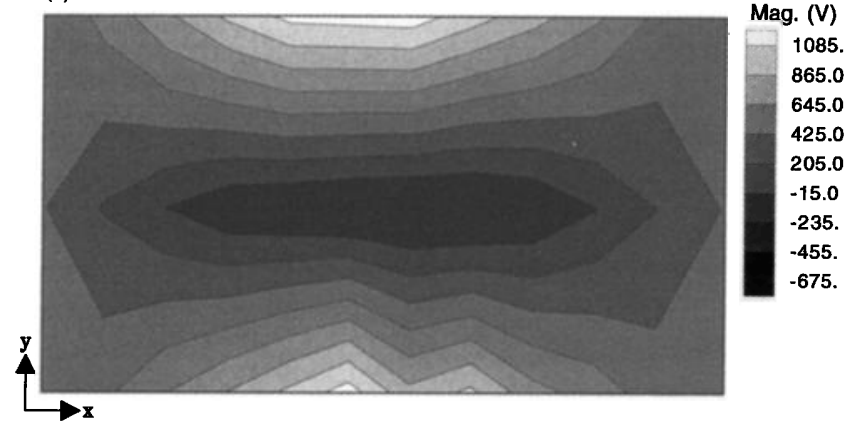

(b)

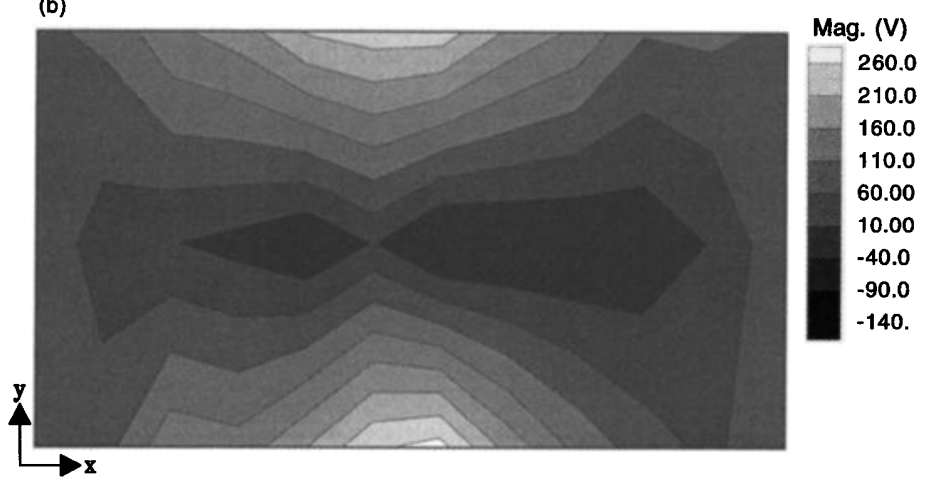

FIG. 6. Receiving plate vibration distribution at $176 \mathrm{~Hz}$. (a) Without control, (b) with control $(\mathrm{S} 1, \mathrm{~S} 2, \mathrm{C} 1, \mathrm{C} 2)$.

$(1,3)$ mode]. As in the previous case at $106 \mathrm{~Hz}$, it was noticed that the curvature of the receiving plate was reduced on the sensor surface, which leads to an attenuation of the PVDF sensor output voltage. This again shows the importance of the choice for the PVDF locations with respect to the actuator positions, because, as noted previously, the output voltage of the PVDF strip can be driven to zero by the control actuator without implying the out-of-plane vibratory motion to be zero. This also shows that as the frequency is increased and the mode shape becomes more complicated, the number of PVDF sensors has to be increased as well in order to provide enough information to the controller about the vibrational distribution of the receiving plate. As a matter of fact, when two control channels are used, as shown in Fig. $6(b)$, high attenuation of vibrations levels $(14 \mathrm{~dB})$ in the receiving structure is obtained with no effect on the top plate vibrations.

The last set of experiments is related to an off-resonance frequency of the system, $85 \mathrm{~Hz}$. At this frequency, the receiving structure response is between its second and third resonance modes. It can be observed in Fig. 7(a) that the second mode is dominant as a nodal line parallel to the clamped edges is located close to the center of the receiving plate. Therefore, the PVDF sensors were chosen to have the same position as in the second set of experiments $(106 \mathrm{~Hz})$, i.e., one sensor on each side of the nodal line. When two control channels are implemented, the output signal from both PVDF sensors is well attenuated (about $15 \mathrm{~dB}$ ). However, the global vibration amplitudes are decreased by only $4 \mathrm{~dB}$. This shows again the disadvantage of PVDF sensors, which may give a low output voltage while the out-of-plane vibra- 


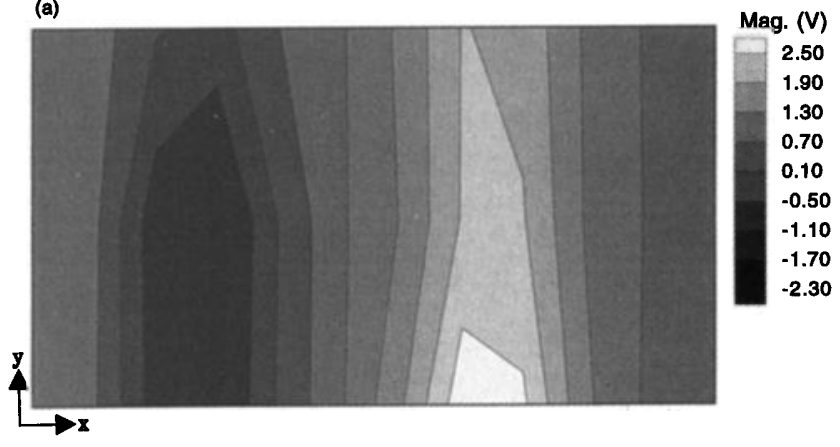

(b)

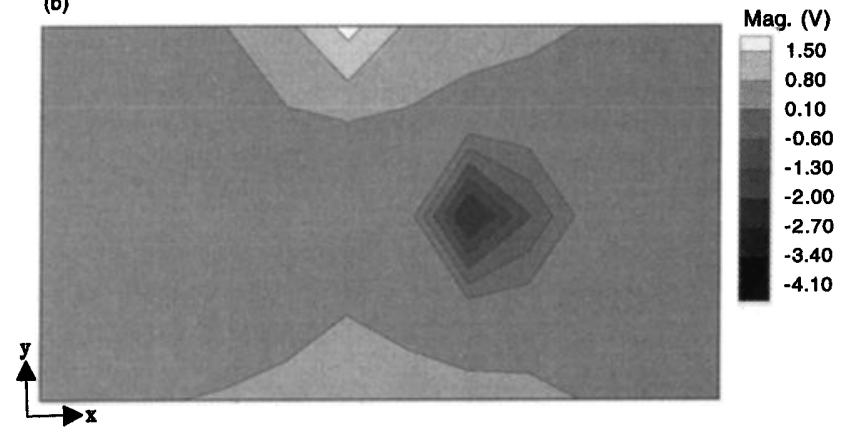

FIG. 7. Receiving plate vibration distribution at $85 \mathrm{~Hz}$. (a) Without control, (b) with control $(\mathrm{S} 1, \mathrm{~S} 2, \mathrm{C} 1, \mathrm{C} 2)$.

tion amplitudes are nonzero. The vibration distribution when the control is applied is presented in Fig. 7(b). It can be seen that the mode shape under control becomes more complex implying contribution from several modes. Two nodal lines corresponding to the diagonals of the receiving plate can be observed. As a result of the location of the nodal line accross the surface covered by each PVDF sensor, the response of both sensors is well attenuated. The amplitudes of the vibrational response under control have been increased in the region where the actuators are located and decreased elsewhere. This phenomenon was also observed in Ref. 8. Then it seems that, in this case, the control of the supporting structure vibration is obtained by modal restructuring, as modal vibration amplitudes are increased in some regions but the phase between the modes induces large attenuation of the sensors output signal. It can be supposed that, in order to obtain larger decrease of the global vibration amplitudes, the number of control channels has to be increased or the position of the sensors and actuators has to be optimized.

\section{CONCLUSIONS}

Active isolation of vibrations transmitted from a rigid machinery raft to a flexible adaptive receiving structure has been experimentally studied. The adaptive process is achieved by distributed piezoelectric actuators and PVDF sensors directly integrated with the receiving structure. Control "intelligence" is provided by an adaptive LMS approach.
The experimental results show that efficient active isolation of vibrations is easily obtained using this technique for on- and off-resonance cases. Large improvement is achieved by increasing the number of PVDF error sensors and the number of piezoelectric actuators used in the control process. The control of the supporting structure vibrations has been obtained by modal reduction, as the modal vibration amplitudes are attenuated in the control process, or by modal restructuring, as the modal amplitudes are increased at some locations but the phase between the modes induces attenuation of the sensors output signal.

In achieving control, strips of PVDF distributed on the surface of the supporting structure were implemented as error sensors. The control effectiveness has shown an important dependance on PVDF sensors, as the output voltage of the PVDF strip can be driven to zero in the control process, even though the out-of-plane vibration amplitudes are not well attenuated or are increased. This problem is avoided by implementing two channels of control (two piezoelectric actuators and two PVDF strips).

As a result, PVDF distributed sensors and piezoelectric actuators show much promise in active isolation of periodic vibrations. Note in the arrangement tested here that control on resonance was achieved with at the most two actuators. By comparison, the conventional approach of active isolation using actuators in parallel/series at each passive mount point would require four channels of control to be effective. Also, the adaptive LMS algorithm provided an effective narrowband controller, which does not require exact system modeling as feedback approaches do.

\section{ACKNOWLEDGMENTS}

The authors gratefully acknowledge the support of this work by NASA Langley and the Office of Naval Research under Grant No. ONR-N00014-88-K-0721. They also wish to thank A. L. Wicks and his students for their valuable help concerning the measurements with the scanning laser vibrometer.

${ }^{1}$ A. H. Von Flotow, "An expository overview of active control of machinery mounts," Proceedings of the 27th Conference on Decision and Control, Austin, Texas, 1988, pp. 2029-2032.

${ }^{2}$ P. A. Nelson, M. D. Jenkins, S. J. Elliott, "Active isolation of periodic vibrations," Noise-Con 87, 425-430 (1987).

${ }^{3}$ S. Lefebvre, C. Guigou, C. R. Fuller, "Experiments on active isolation using distributed PVDF error sensors," J. Sound Vib. 155, 177-184 (1992).

${ }^{4}$ E. K. Dimitriadis, C. R. Fuller, and C. A. Rogers, "Piezoelectric actuators for distributed vibration excitation of thin plates," J. Vib. Acoust. 113, 110-107 (1991).

${ }^{5}$ C. K. Lee and F. C. Moon, "Modal sensors/actuators," J. Appl. Mech. 57, 434-441 (1990).

${ }^{6}$ S. J. Elliott, I. M. Stothers, and P. A. Nelson, "A multiple error LMS algorithm and its application to the active control of sound and vibration," IEEE Trans. Acoust. Speech Signal Process. ASSP-35, 1423-1434 (1987).

${ }^{7}$ A. W. Leissa, "Vibrations of plates," NASA SP-160 (1969).

${ }^{8} \mathrm{~S}$. Lefebvre, "Active control of interior noise using piezoelectric actuators in a large-scale composite fuselage model, M.S. thesis, Virginia Polytechnic Institute and State University (1991). 